\title{
Article
}

\section{Epigenetic Influences on Neurodevelopment at 11 Years of Age: Protocol for the Longitudinal Peri/Postnatal Epigenetic Twins Study at 11 Years of Age (PETS@11)}

\author{
Pamela Leong ${ }^{1,2}$, Yuk Jing Loke ${ }^{1,2}$, Richard Saffery ${ }^{1,2}$, Tim Silk ${ }^{3,4}$, Alice Burnett ${ }^{2,5,6,7}$, Elisha Josev ${ }^{1,2,8}$, Katrina Scurrah ${ }^{9}$, \\ Christiane Theda ${ }^{1,2,10}$, Anna Czajko ${ }^{1}$, Emmanuel PK Pua ${ }^{4,11}$, Marc L Seal ${ }^{2,4}$ and Jeffrey M Craig M $^{1,2,12}$ \\ ${ }^{1}$ Epigenetics Group, Murdoch Children's Research Institute, Melbourne, Victoria, Australia, ${ }^{2}$ Department of Paediatrics, The University of Melbourne, Melbourne, \\ Victoria, Australia, ${ }^{3}$ School of Psychology, Deakin University, Melbourne, Victoria, Australia, ${ }^{4}$ Developmental Imaging Group, Murdoch Children's Research \\ Institute, Melbourne, Victoria, Australia, ${ }^{5}$ Victorian Infant Brain Studies Group, Murdoch Children's Research Institute, Melbourne, Victoria, Australia, ${ }^{6}$ Premature \\ Infant Follow-Up Programme, Royal Women's Hospital, Melbourne, Victoria, Australia, ${ }^{7}$ Neonatal Medicine, Royal Children's Hospital, Melbourne, Victoria, \\ Australia, ${ }^{8}$ Neurodisability and Rehabilitation Group, Murdoch Children's Research Institute, Melbourne, Victoria, Australia, ${ }^{9}$ Twins Research Australia, Centre for \\ Epidemiology and Biostatistics, Melbourne School of Population and Global Health, The University of Melbourne, Melbourne, Victoria, Australia, ${ }^{10}$ Obstetrics and \\ Gynaecology, Royal Women's Hospital, Melbourne, Victoria, Australia, ${ }^{11}$ Melbourne School of Psychological Sciences, University of Melbourne, Melbourne, \\ Victoria, Australia and ${ }^{12}$ Centre for Molecular and Medical Research, Deakin University School of Medicine, Geelong, Victoria, Australia
}

\begin{abstract}
Neurodevelopment is sensitive to genetic and pre/postnatal environmental influences. These effects are likely mediated by epigenetic factors, yet current knowledge is limited. Longitudinal twin studies can delineate the link between genetic and environmental factors, epigenetic state at birth and neurodevelopment later in childhood. Building upon our study of the Peri/postnatal Epigenetic Twin Study (PETS) from gestation to 6 years of age, here we describe the PETS 11-year follow-up in which we will use neuroimaging and cognitive testing to examine the relationship between early-life environment, epigenetics and neurocognitive outcomes in mid-childhood. Using a within-pair twin model, the primary aims are to (1) identify early-life epigenetic correlates of neurocognitive outcomes; (2) determine the developmental stability of epigenetic effects and (3) identify modifiable environmental risk factors. Secondary aims are to identify factors influencing gut microbiota between 6 and 11 years of age to investigate links between gut microbiota and neurodevelopmental outcomes in mid-childhood. Approximately 210 twin pairs will undergo an assessment at 11 years of age. This includes a direct child cognitive assessment, multimodal magnetic resonance imaging, biological sampling, anthropometric measurements and a range of questionnaires on health and development, behavior, dietary habits and sleeping patterns. Data from complementary data sources, including the National Assessment Program - Literacy and Numeracy and the Australian Early Development Census, will also be sought. Following on from our previous focus on relationships between growth, cardiovascular health and oral health, this next phase of PETS will significantly advance our understanding of the environmental interactions that shape the developing brain.
\end{abstract}

Keywords: longitudinal; epigenetics; neurodevelopment; prenatal; postnatal; adolescence; twins

(Received 27 November 2019; accepted 28 November 2019)

Neurodevelopment, which is influenced by genetic, environmental and stochastic factors, has a significant impact on later cognitive, motor, language and behavioral functions (Bick \& Nelson, 2016; Chiarella et al., 2015). However, the relative contributions of these factors to neurodevelopmental outcomes are unclear.

At the interface of genes and environment, epigenetic variation is thought to mediate many early-life developmental changes (Chiarella et al., 2015; Petanjek \& Kostovic, 2012), although disentangling the relative contributions of genetic versus environmental influence is problematic in humans. Twin studies represent a

Author for correspondence: Jeffrey M Craig, Email: Jeffrey.craig@deakin.edu.au Cite this article: Leong P, Loke YJ, Saffery R, Silk T, Burnett A, Josev E, Scurrah K, Theda C, Czajko A, Pua EPK, Seal ML, and Craig JM. (2019) Epigenetic Influences on Neurodevelopment at 11 Years of Age: Protocol for the Longitudinal Peri/Postnatal Epigenetic Twins Study at 11 Years of Age (PETS@11). Twin Research and Human Genetics 22: 446-453, https://doi.org/10.1017/thg.2019.120 powerful approach to understand the relative contributions of genetic variation and shared and nonshared environments to any phenotype, including neurodevelopmental outcomes in children. Within-pair modeling controls for shared and potentially unmeasured genetic and environmental factors, which may confound associations observed in nontwin studies.

The Peri/postnatal Epigenetic Twins Study (PETS) study began in 2006 with the recruitment of women who were pregnant with twins from three major maternity hospitals in Melbourne, Australia (Saffery et al., 2012). A total of 251 women and their twins were recruited between 2007 and 2009. Data and biological samples were collected from the mothers and their infants at four time points: during the pregnancy, at the time of the twins' births, and again at both 18 months and 6 years of age. Previous sample and data collection methods to 18 months of age have been previously published (Loke, Novakovic et al., 2013; Saffery et al., 2012) 
and are summarized in Table 1, along with data and sample collection at 6 years of age (manuscript in preparation).

Briefly, data collected from the time of recruitment and during pregnancy included information regarding sociodemographic characteristics, mode of conception, ultrasound scans, and maternal health and dietary intake. In addition, maternal serum was collected at 28 weeks' gestation. Most of the births were attended by a researcher (75\%), and of these, a range of biological samples and neonatal information was collected (91\%). This included buccal (cheek) swabs, placental tissue, and cord blood and cord tissue, from which purified umbilical vein endothelial cells were isolated, as well as neonatal information regarding birth mode, placental weight, cord insertion and anthropometric measurements. When the twins reached 18 months of age, families were contacted again in order to collect further information about the twins' health and development, their diet, anthropometry and biological samples (e.g., buccal swabs and venous blood; Loke, Novakovic et al., 2013; Temples et al., 2016).

At 6 years of age, the primary focus was to explore the links between oral health, heart health and gut health in the twins. Biological samples (fecal samples, saliva samples and dental plaque) and information regarding the twins' health, development and diet were collected. The twins also underwent dental examinations and completed an additional questionnaire regarding their oral health and dental health practices (M. Silva et al., 2019; M. J. Silva et al., 2019).

To date, PETS has enabled an increased understanding of the influence of environment on gene activity via epigenetics in early life. For example, we found that genetically identical twins can differ in their epigenetic mark of DNA methylation at birth (Gordon et al., 2012; Li et al., 2017; Li et al., 2018; Ollikainen et al., 2010; Saffery et al., 2012). Understanding of this effect of the 'nonshared' environment is likely to contribute toward understanding of how experiences in the womb may affect later-life health outcomes (Heijmans et al., 2008; Roseboom et al., 2006; Veenendaal et al., 2012). This information is also invaluable in understanding how early-life events are associated with differences in neurodevelopment in mid-childhood, which is the current focus of PETS.

\section{Aims}

The principle aim for the current wave is to assess within-twin-pair differences in early-life DNA methylation state and to identify early-life events that are associated with these and/or differences in neurodevelopmental outcomes in mid-childhood. The specific aims are to (1) identify which epigenetic markers (at birth) are associated with cognitive and behavioral outcomes at age 11 to contribute to the development of early predictive biomarkers; (2) assess the developmental stability of epigenetic markers from birth to age 11 to inform whether the same or different biomarkers will be needed at different ages and (3) identify specific nonshared intrauterine exposures associated with epigenetic marks at birth, which could represent potentially modifiable intervention targets.

Secondary aims include identifying factors that influence the gut microbiota between the ages of 6 and 11 years and to investigate the links between gut microbiota and neurodevelopmental outcomes at age 11 years.

\section{Methods}

\section{Study Design and Participants}

Ethical approval to undertake the current wave was granted by the Royal Children's Hospital (RCH), Melbourne (HREC 38018). By the end of the 6 -year wave, 216 twin pairs $(85 \%$ of the original cohort) remained in the study. Of these, 85 (40\%) were monozygotic (MZ), 66 (30\%) were same-sex dizygotic and the remaining $65 \mathrm{DZ}$ twin pairs were of opposite sex (30\%). The attrition rate was less than $2 \%$ per year. The current wave will include core sample and data collection components, such as buccal swabs, anthropometric measurements, cognitive assessments and questionnaires, as well as optional components such as providing blood samples, fecal samples and magnetic resonance imaging (MRI) brain scans.

\section{Inclusion and Exclusion Criteria}

All 216 twin pairs who remained in the study following the 6-year wave of data collection are currently being invited to participate as they approach 11 years of age ( \pm 3 months). The 151 same-sex twin pairs are offered all measures, including optional MRI brain scans. Due to the focus on controlling for sex in the study, opposite-sex twin pairs will not undergo an MRI but will be invited to participate in all other aspects of the study. Children with known congenital brain malformations will also not undergo an MRI due to the limited comparability of these scans with unaffected children. Finally, children are excluded from participating in the MRI component of the study if there are known contraindications to the MRI scan, as determined by the attending staff of the Royal Children's Hospital Medical Imaging Team. These include metal implants from surgery (e.g., pacemakers and cardiac clips), presence of metallic foreign bodies, metallic implants that elicit severe image artifact (e.g., orthodontic banding) or severe neurosensory impairments.

\section{Procedure}

Participation involves a single visit to the Murdoch Children's Research Institute at the Royal Children's Hospital in Melbourne. Briefly, parents complete five online questionnaires prior to attending. Children are also asked to complete two questionnaires each. The study visit takes between 4 and $6 \mathrm{~h}$, depending on whether families consent to optional components of the study and meet the inclusion criteria for the optional MRI (Figure 1). Direct assessments are conducted by trained research psychologists and research assistants. Biological samples (saliva and buccal swabs) and anthropometric measurements are collected by the research coordinator or a trained research assistant.

In addition to the core components of the study, additional components include a brain MRI scan, collection of a venous blood sample and collection of a fecal sample. MRI data are acquired by the Royal Children's Hospital Medical Imaging staff. Parents may also provide consent for the study team to link data from complementary data sources, including Medicare and Pharmaceutical Benefit Scheme (PBS), National Assessment Program - Literacy and Numeracy (NAPLAN), Australian Early Development Census (AEDC) and the School Entrant Health Questionnaire (SEHQ). This additional data will provide important information about health service use as well as classroom behavior and learning.

\section{Core Data Collection}

\section{Questionnaires.}

Health and development at 11 years. This questionnaire has been developed by the study team to obtain key health and developmental information including some items that are consistent with previous waves, allowing for comparisons and analysis of health and development longitudinally. The questionnaire has 20 items seeking parental opinions about their child's development in the 
Table 1. Summary of sample and data collection

\begin{tabular}{|c|c|c|c|c|c|}
\hline \multicolumn{6}{|l|}{ Gestation to birth } \\
\hline Parental data and samples ${ }^{\mathrm{a}}$ & $18-20$ weeks & 24 weeks & 28 weeks & 36 weeks & $\begin{array}{l}\text { Birth/immediate } \\
\text { neonatal period }\end{array}$ \\
\hline Height & C & & & & \\
\hline Weight & & C & & C & \\
\hline Age & c & & & & \\
\hline Education & c & & & & \\
\hline Sociodemographics & c & & & & \\
\hline Past medical and obstetric history & C & & & & \\
\hline Current medical and obstetric history & c & C & & c & \\
\hline Mode of conception & c & & & & \\
\hline Nutritional supplements & c & C & & c & \\
\hline Medication & c & C & & c & \\
\hline Alcohol intake & C & c & & c & \\
\hline Smoking & C & c & & C & \\
\hline Food frequency questionnaire & & & C & & \\
\hline Perceived Stress Questionnaire & & & c & & \\
\hline Peripheral blood sample & & & c & & \\
\hline Placental examination & & & & & C \\
\hline Cord blood sample/Guthrie cards & & & & & c \\
\hline Umbilical cord sample & & & & & $\mathrm{C}$ \\
\hline Placental tissue sample & & & & & C \\
\hline Labor and delivery data & & & & & c \\
\hline Ultrasound data (all possible time points) & C & C & c & c & \\
\hline Child data and samples & $\begin{array}{l}\text { Birth/im } \\
\text { neonatc } \\
\quad 18 \mathrm{mc}\end{array}$ & $\begin{array}{l}\text { diate } \\
\text { eriod } \\
s^{\mathrm{a}}\end{array}$ & 18 months $^{\mathrm{a}}$ & 6 years & 11 years \\
\hline Time and date of birth & & & & & \\
\hline Requirement for specialist/intensive care & & & & & \\
\hline Ultrasound data & & & & & \\
\hline Anthropometric measures & & & c & C & C \\
\hline Breastfeeding history ${ }^{\mathrm{b}}$ & & & c & & \\
\hline Nutritional history/diet ${ }^{\mathrm{b}}$ & & & c & c & C \\
\hline Supplements ${ }^{\mathrm{b}}$ & & & c & c & C \\
\hline Illnesses and allergies ${ }^{\mathrm{b}}$ & & & C & C & C \\
\hline Health and development ${ }^{\mathrm{b}}$ & & & C & C & C \\
\hline World View and Empathy ${ }^{c}$ & & & & & C \\
\hline Household smoking ${ }^{\mathrm{b}}$ & & & c & c & C \\
\hline Dental/oral care and practices history ${ }^{b}$ & & & & c & \\
\hline Oral examination & & & & C & \\
\hline Peripheral blood & & & 0 & 0 & 0 \\
\hline Buccal swab samples & & & $c$ & c & C \\
\hline Saliva samples & & & & C & C \\
\hline Dental plaque samples & & & & C & \\
\hline Fecal samples & & & & 0 & 0 \\
\hline Cognitive function assessments & & & & & c \\
\hline MRI & & & & & 0 \\
\hline
\end{tabular}

Note: C, Core data/sample collection; O, Optional data/sample collection.

adapted from Loke, Novakovic, et al. (2013) and Saffery et al. (2012).

bParentally reported either as stand-alone questionnaire or contained in questionnaires.

${ }^{\mathrm{c}}$ Child reported/completed. 
Fig. 1. Outline of a typical schedule of a study visit day. The start time of the study visit is dependent on the type of consent given, but variability is minimized (within an hour difference) to ensure time of sample and data collection are consistent across all participants. The twins' order of MRI and cognitive assessment data acquisition will be randomized to ensure balanced proportion of first-born and secondborn completing the cognitive protocol prior to MRI and vice versa. Note: *Actual start and finish times will vary depending on room availability, participant preference (including breaks) and type of consent.

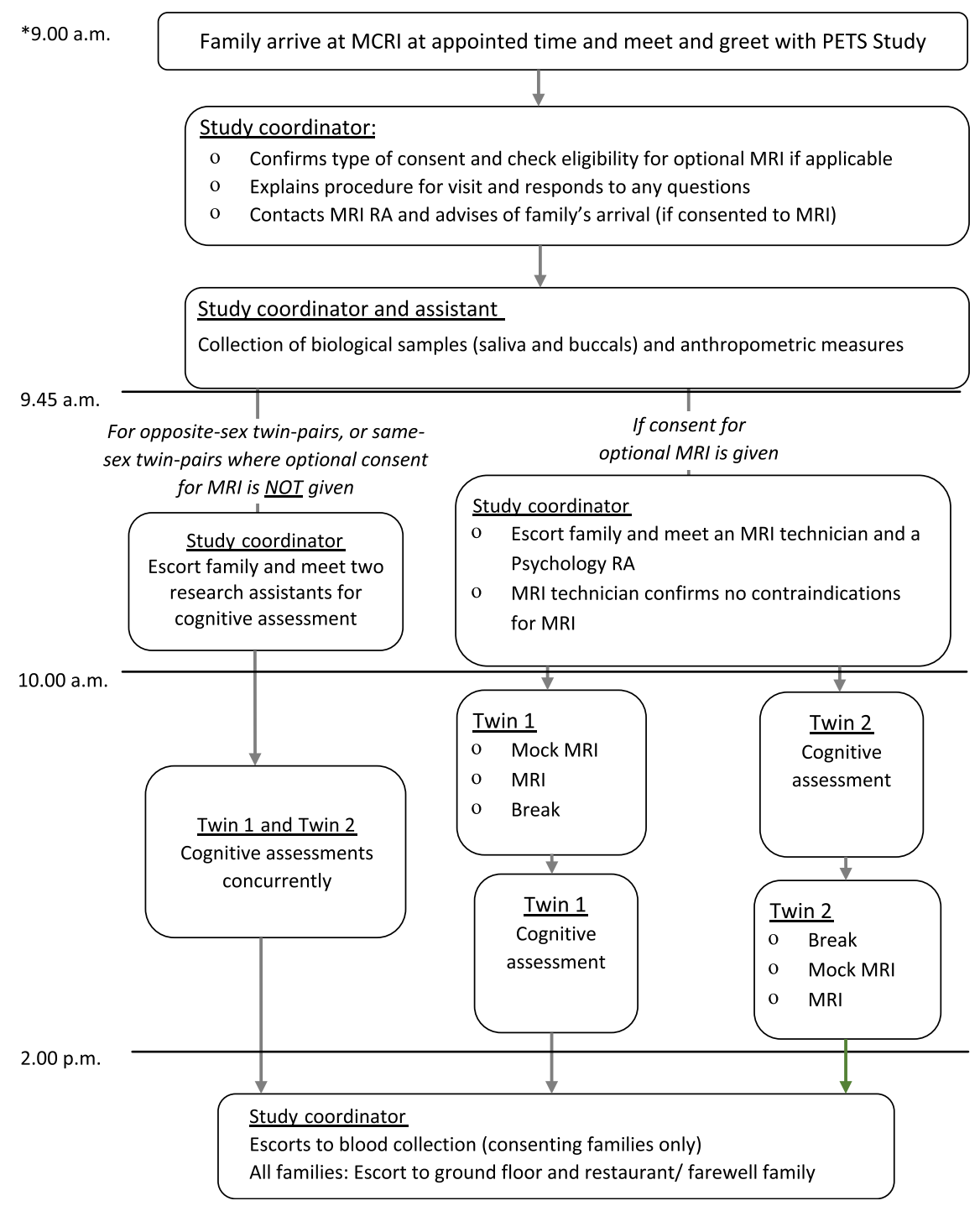

broad areas of language, vision, understanding and physical coordination. Parents are also asked about medically diagnosed conditions or syndromes, presence of any illnesses, infections, accidents or allergies and whether the twins have been exposed to smoking in the home.

Diet and dietary habits at 11 years. This questionnaire was developed for use in previous waves of PETS and has been modified to enable longitudinal analyses as well as to accommodate the increased age of the children. The questionnaire consists of 40 items and asks about the variety and frequency of intake of various food items from a range of fruit and vegetables, dairy, meat and other proteins that are consumed by the twins in the past month as well as in the past week. In addition, information is sought about water, juice and other fluid intake, as well as the consumption of sugars either added to foods or contained in processed foods such as breakfast cereals.

Environmental worldviews (Manoli et al., 2007). The 10-item New Ecological Paradigm scale for children is a validated questionnaire and will be completed by each twin independently. It measures affective, cognitive and behavioral aspects of how the individual feels about the natural world. The questionnaire has a total of 10 questions with responses entered using a 5-point Likert-type scale.

Measuring empathy (Garton \& Gringart, 2005). The two-factor structure solution of the 'Feeling and Thinking' instrument is a 12 -item validated questionnaire that is new to the twins and will be completed by each twin working independently. It measures their competence at relating to others and their predisposition to establish and maintain joint communication and sharing, which has been related to environmental worldviews (Metz, 2017). There are 12 questions in total with responses entered on a 5-point Likert-type scale.

Sleep patterns (Quach et al., 2009; Sung et al., 2008). This parent-reported and validated questionnaire consists of a total of two items requiring multiple tick-a-box responses.

Pubertal Development Scale (Petersen et al., 1988). The Pubertal Development Scale is a validated parent-reported measure assessing development on five indices of pubertal growth. There are eight items on a 4-point scale.

Behavior Assessment System for Children - Third Edition (Reynolds \& Kamphaus, 2015). This standardized questionnaire 
asks parents to rate how often their child displays various behaviors and emotions to assess behavioral and emotional wellbeing.

\section{Anthropometric Assessments}

Body measurements including head, upper left arm and abdominal circumferences, blood pressure, height, weight, triceps and scapular skin-fold thickness and bioimpedance are taken by the research coordinator or a trained and calibrated research assistant and recorded directly onto an electronic form at the time of the visit.

\section{Biological Sample Collection}

Biological samples are deidentified on collection and coded with a unique study identification number. The codes are reidentifiable by using a master file stored on a password-protected computer hard drive.

Saliva samples are collected by the research coordinator or a trained research assistant at the start of the visit. Approximately $1 \mathrm{~mL}$ will be collected by passive drool. Following collection, the saliva is placed on wet ice and transported to the laboratory. On the same day, an amount of $200 \mu \mathrm{L}$ of the saliva sample will be mixed with $800 \mu \mathrm{L}$ TRIzol reagent (Ambion, TX, USA) and then equally divided between two FluidX $.8 \mathrm{~mL}$ tubes (Brooks Life Science, MA, USA) for storage. The remaining saliva will be then aliquoted into microcentrifuge tubes in amounts of $250 \mu \mathrm{L}$ for storage.

Buccal swabs (cheek cell samples) are collected by the research coordinator following the collection of saliva samples. Three swabs (left cheek, right cheek and both cheeks) are collected from each child using COPAN FLOQ swabs as previously described (Saffery et al., 2012). The buccal swabs obtained from the left and right cheeks, respectively, will be twizzled into $1 \mathrm{~mL}$ RNA Protect Cell reagent (Qiagen, Venlo, the Netherlands) and then stored in $500-\mu \mathrm{L}$ aliquots at $-80^{\circ} \mathrm{C}$. The third swab (both cheeks) is resheathed for storage.

\section{Cognitive Assessments}

The Wechsler Intelligence Scale for Children - Fifth Edition, Australian and New Zealand Standardised Edition (WISC-V; Wechsler, 2016) is a gold-standard measure of intellectual functioning in children. The 10 primary subtests of the WISC-V will be administered via Q-interactive, a secure digital platform.

Word reading, mathematical computation and spelling abilities will be measured using the Wide Range Achievement Test, Fifth Edition (Wilkinson \& Robertson, 2017).

The NIH Toolbox for Assessment of Neurological and Behavioral Function (Gershon et al., 2013) provides customizable computerized tests in a wide range of cognitive domains. Four age-appropriate tasks have been selected to assess attention and executive function (Inhibitory Control and Attention Test, Dimensional Change and Card Sort Test), memory (Picture Sequence Memory Test) and processing speed (Pattern Comparison Processing Speed Test).

For twin pairs also completing the optional MRI component (below), the order of acquisition of cognitive and MRI data will be randomized such that equal numbers of first-born and secondborn twins will complete the cognitive protocol prior to imaging and vice versa.

\section{Data Collection: Optional}

Magnetic resonance imaging. MRI is highly challenging in pediatric cohorts due to issues with compliance or entering the scanner and head-motion movement artifacts that can severely impact the quality of image acquisition (Makowski et al., 2019). Prior to the MRI, children complete a prescan MRI preparation protocol involving a 30-min session in a mock scanner with a trained research psychologist or research assistant to familiarize themselves with the MRI environment and reduce anxiety and likelihood of distress or movement during scanning as well as associated motion-related artifacts (for description of mock MRIsimulation procedure, see Pua et al., 2019).

MRI data are collected from a single, research-dedicated 3-Tesla MAGNETOM Prisma system (Siemens Medical Systems, Erlangen, Germany) equipped with a 32-channel phased-array head coil. The proposed MRI protocol has been optimized for pediatric participants and comprises of structural and functional sequences lasting approximately $45 \mathrm{~min}$ (see sequences details in Table 2).

Structural imaging. To measure brain structure, a T1-weighted multiecho magnetization-prepared rapid gradient-echo sequence is acquired, optimized for dealing with head motion. This will provide high-resolution anatomical images for tissue segmentation and estimates of cortical thickness and surface area, which will be performed with the FreeSurfer image analysis suite.

Diffusion-weighted imaging. Diffusion-weighted images will be acquired to examine the physical white matter connections (structural connectivity). A multishell protocol $\left(b=3000,2000,1000 \mathrm{~s} / \mathrm{mm}^{2}+\right.$ interleaved $b=0 \mathrm{~s} / \mathrm{mm}^{2}$ ) is acquired in an anterior-posterior phase encoding direction, with Blip Up and Blip Down sequences to correct for magnetic susceptibility-induced distortions. High angular resolution diffusion imaging will be used for spherical deconvolution tractography to map structural connectivity.

Resting-state functional MRI. Resting-state fMRI images are acquired to measure spontaneous intrinsic neural activity (functional connectivity) between cortical and subcortical regions. Participants are asked to keep eyes open and to focus on a fixation cross that is projected on to a screen, for the $5 \mathrm{~min} 46 \mathrm{~s}$ sequence.

Fecal samples. Parents who consent to providing the twins' fecal samples will be mailed a kit with instructions for collection, storage and transport to $\mathrm{RCH}$. The kit contains plastic containers, biohazard bags, prelabeled fecal containers (Sarstedt, Nümbrecht, Germany), disposable gloves, ice pack and freezer bag. The child is asked to first urinate in the toilet bowl before collecting stool sample to prevent contamination of the stool sample with urine. Once the child deposits the stool directly into the plastic container, the stool is collected (near the middle rather than the edges of the sample) using the 'spoon' attached to the lid of feces container. Once the tube is filled and closed, it is placed in the specimen bag and then stored in home freezer immediately with the ice pack. On the day of the study visit, the family place the frozen sample and ice pack in the given freezer bag and bring to the study visit. On the same day of the visit, the fecal samples are aliquoted into $2-\mathrm{mL}$ tubes and stored at $-80^{\circ} \mathrm{C}$.

Blood samples. Blood samples are collected by a phlebotomist at the end of the study visit. Twins who provide these samples will have no more than $9 \mathrm{~mL}$ venous blood collected for processing, including blood counts and isolation of plasma and peripheral blood mononuclear cells for cryopreservation at $-80^{\circ} \mathrm{C}$ and in liquid nitrogen, respectively. The tubes will be spun at $500 \mathrm{~g}$ for $10 \mathrm{~min}$ at $20^{\circ} \mathrm{C}$ with no brake and full acceleration. Plasma is 
Table 2. MRI sequence parameters for scanning

\begin{tabular}{|c|c|c|c|c|c|c|c|c|c|}
\hline \multirow{2}{*}{$\begin{array}{l}\text { Sequence } \\
\\
\text { Type }\end{array}$} & \multirow{2}{*}{$\begin{array}{c}\text { T1w } \\
\text { ME-MPRAGE }\end{array}$} & \multirow{2}{*}{$\frac{\mathrm{T} 2 \mathrm{w}}{}$} & \multicolumn{4}{|c|}{ DWI } & \multicolumn{2}{|c|}{ fMRI } & \multirow{2}{*}{$\begin{array}{c}\text { T2 } \\
\text { T2 } \\
\text { CS-Space }\end{array}$} \\
\hline & & & Shell 1 & Shell 2 & Shell 3 & $\begin{array}{l}\text { Reverse } \\
\text { phase- } \\
\text { encoding } \\
\text { Blips }\end{array}$ & rs-fMRI & $\begin{array}{l}\text { Reverse } \\
\text { phase- } \\
\text { encoding } \\
\text { Blips }\end{array}$ & \\
\hline $\mathrm{TR}(\mathrm{ms})$ & 2550 & 3200 & 3500 & 3500 & 3500 & 3570 & 800 & 800 & 3200 \\
\hline $\mathrm{TE}(\mathrm{ms})$ & $2.14,3.945 .77,7.5$ & 563 & 74.4 & 74.4 & 74.4 & $73 / 76$ & 37 & 66 & 563 \\
\hline $\mathrm{TI}(\mathrm{ms})$ & 1550 & - & - & - & - & - & - & - & - \\
\hline Flip angle (deg) & 8 & - & 90 & 90 & 90 & 90 & 52 & 90 & - \\
\hline Slices & 208 & 208 & 66 & 66 & 66 & 66 & 72 & 72 & 208 \\
\hline Voxel size (mm) & $.4 \times .4 \times .9$ & $.8^{3}$ & $2^{3}$ & $2^{3}$ & $2^{3}$ & $2^{3}$ & $2^{3}$ & $2^{3}$ & $.8^{3}$ \\
\hline FoV read $(\mathrm{mm})$ & 256 & 256 & 220 & 220 & 220 & 220 & 208 & 208 & 256 \\
\hline FoV phase (\%) & 100 & 93.8 & 100 & 100 & 100 & 100 & 100 & 100 & 93.8 \\
\hline Matrix & $640 \times 640$ & $320 \times 300$ & $110 \times 110$ & $110 \times 110$ & $110 \times 110$ & $110 \times 110$ & $104 \times 104$ & $104 \times 104$ & $320 \times 320$ \\
\hline Band width (Hz/Px) & $790,670,720,790$ & 744 & 2272 & 2272 & 2272 & 2272 & 2290 & 2290 & 744 \\
\hline Echo spacing (ms) & 9.5 & 3.52 & .53 & .53 & .53 & .53 & .58 & .58 & 3.52 \\
\hline Orientation & $\mathrm{S}$ & $\mathrm{S}$ & $\mathrm{T}$ & $\mathrm{T}$ & $\mathrm{T}$ & $\mathrm{T}$ & $\mathrm{T}$ & $\mathrm{T}$ & $\mathrm{T}$ \\
\hline$B$ value $\left(\mathrm{s} / \mathrm{mm}^{2}\right)$ & - & - & 3000 & 2000 & 1000 & 0 & - & - & - \\
\hline No. directions $/ b=0 \mathrm{~s}$ & - & - & $64 / 6$ & $45 / 6$ & $25 / 6$ & -12 & - & - & - \\
\hline Multiband factor & - & - & 2 & 2 & 2 & 2 & 8 & 1 & - \\
\hline Acquisition time & $6 \min 47 s$ & $5 \min 57 s$ & $4 \min 21 \mathrm{~s}$ & $3 \min 14 s$ & $2 \min 04$ & $46 s(\times 2)$ & $5 \min 46 s$ & $32 \mathrm{~s}(\times 2)$ & $5 \min 57 s$ \\
\hline
\end{tabular}

Note: S, sagittal; T, transverse; DWI, diffusion-weighted imaging; ME-MPRAGE, multieco magnetization-prepared rapid gradient-echo; T1w, T1-weighted; T2w, T2-weighted; CS-SPACE, 3D Sampling perfection with application optimized contrasts using different flip angle evolution; TR, Repetition time; TE, Echo time; TI, inversion time; FoV, Field of View.

removed and aliquots of $500 \mu \mathrm{L}$ will be stored at $-80^{\circ} \mathrm{C}$. The mononuclear cells and granulocytes will be isolated using Ficoll and Paque PLUS and mixed with fetal bovine serum, 10\% dimethyl sulfoxide and aliquoted into $500-\mu \mathrm{L}$ barcoded cryotubes. The tubes are then frozen at a controlled rate (decrease of $1{ }^{\circ} \mathrm{C} / \mathrm{min}$ ) and stored in liquid nitrogen.

\section{Data Linkage}

Optional consent will also be sought for linkage to additional preexisting administrative databases to obtain additional information about early-child development, health service use and academic performance. Sources include Medicare, PBS, AEDC, SEHQ and NAPLAN. Medicare and the PBS provide important information regarding health service use. AEDC, which is measured by the teacher in the first year of school, measures five domains of childhood development: physical health and wellbeing, social competence, emotional maturity, school-based language and cognitive skills, and communication skills and general knowledge. The SEHQ is a parent report about their concerns regarding their child's health and wellbeing as they commence primary school. NAPLAN, the national standardized academic testing, assesses six domains: reading, writing, spelling, grammar and punctuation, and numeracy.

\section{Statistical Methods}

To address Aim 1, we will use within-twin-pair regression models (Carlin et al., 2005) to quantify the associations between withinpair differences in DNA methylation state in buccal samples at birth and within-pair differences in the outcomes: (1) cognitive and behavioral functioning and (2) brain structure and function at age 11 years. These models, which are linear regression models without an intercept, also allow adjustment for factors that potentially differ between twins in a pair. To additionally allow adjustment for measured factors that are shared between twins in a pair, we will stratify analyses (where sample sizes within each stratum are sufficiently large) or fit within-between models (Carlin et al., 2005). We will first analyze whether potential technical (e.g., array) and biological confounders (e.g., maternal smoking, birth weight, gestational age and cellular heterogeneity) are associated with the DNA methylation data using multidimensional scaling and correlation analysis. Results from this analysis will dictate whether we fit regression models, including confounding factors, or use a novel technique for removal of unwanted technical and biological variation, which was shown to be very effective even on noisy Epigenome-Wide Association Studies data with a weak signal (Maksimovic et al., 2015). Results will be corrected for multiple testing using the Benjamini Hochberg false discovery rate method (Benjamini \& Hochberg, 1995). This will yield a list of differentially methylated probes (DMPs) ranked by false discovery rate values. Differentially methylated regions (DMRs) will be determined using the BumpHunter package (Jaffe et al., 2012) for Bioconductor. For DMP/DMR-associated genes, we will perform gene ontology and pathway analyses using methods developed for Infinium methylation data from the missMethyl package (Phipson et al., 2016). In addition to standard single-marker approaches, we will apply data-reduction methods, including machine learning and estimation of cumulative methylation risk scores (Cecil et al., 2016) and DNA methylation ages, to enable assessment of association of groups of epigenetic markers with neurocognitive outcomes.

To determine the developmental stability of epigenetic effects (Aim 2), we will conduct a longitudinal analysis of DNA methylation from buccal samples. Within twin pairs, we will identify whether DNA methylation level of genes identified at birth for each 
11-year outcome in Aim 1 is stable in their association when DNA methylation is measured at 18 months, 6 years and 11 years of age. We will fit similar models to those described for Aim 1 to assess whether within-pair differences in genomewide DNA methylation at the three additional time points are associated with within-pair differences in the neurocognitive outcomes described above. If the longitudinal, within-pair associations do not hold for any time point, we will determine those that do, using the same method.

To identify specific intrauterine exposures as potentially modifiable intervention targets (Aim 3), linear regression models will be fitted to quantify associations of within-pair differences in the following twin-specific exposure variables with within-pair differences in DNA methylation risk score at birth.

- Birth weight relative to gestational age, expressed as standard deviation score (Levesque et al., 2015)

- Head growth rate between 20 and 35 weeks' gestation: from head circumference from four longitudinal ultrasounds

- Levels of clinical and subclinical inflammation at birth: from C-reactive protein in cord blood serum. Previously associated with neurodevelopmental outcomes in singletons (Hagberg et al., 2012)

- Placenta weight: for the $40 \%$ of MZ twins with two placentas and all DZ twins

- Average umbilical artery flow rate from 20 to 35 weeks: from ultrasound Doppler measurements

- Point of umbilical cord insertion into the placenta (Loke, Galati et al., 2013)

- Apgar score: a summary measure of neonatal condition immediately after birth

- Length of stay in the neonatal intensive care unit: proportional to morbidity in the first weeks of life

Analyses may be stratified by postnatal variables shared within twin pairs including sex, gestational age and socioeconomic status, or within-between models will be fitted (Carlin et al., 2005), as appropriate. As most of our predictor variables are associated with fetal growth, we will test for collinearity and eliminate those shown to be dependent and correlated.

\section{Strengths and Weaknesses of the Study}

This is a longitudinal twin study of an established cohort with comprehensive prospective data collection by an established research group.

Specifically, this longitudinal study now encompasses seven time points commencing in the second trimester of pregnancy and following the participants and their families for over a decade. The low attrition rate is noteworthy. In Australia, it is possible to further complement health data obtained at the study visit with data from the public health care system and testing through the educational system. The evaluation at the 11-year study visit is multidisciplinary and comprehensive and performed by relevant experts in their respective fields. The multiple biological samples collected at the multiple time points enable longitudinal epigenetic studies that will be most informative to investigate epigenetic regulation and drift over time and explore their correlations with functional and structural neurodevelopment; the twin study design with the incorporation of within-pair analysis adds further strength.

The sample size is limited to offer participation to the 216 twin pairs who remained in the study at 6 years; MRI scans will be offered to 151 same-sex twin pairs. While this will provide a sufficient sample size for many variables examined, it might not provide sufficient power for analysis or others, thus constituting a weakness for some aspects of our study.

Limited to a single day for evaluation, some tests might be affected by participant fatigue; however, we have gone to great efforts though to structure the study visit to avoid children becoming overwhelmed and to minimize fatigue. As all biological samples are collected on the same day the neurodevelopmental testing and imaging are done, we are unable to fast participants or standardize diets prior to sample collection so as not to overburden the children and families; this may be considered a weakness of our protocol.

\section{Conclusions}

The PETS 11-year follow-up study protocol provides a comprehensive evaluation of functional and structural neurodevelopment combined with assessment of epigenetics and gut microbiome for a substantial number of twin participants. The prospective antenatal recruitment, sample and data collections over a decade ago and multiple assessments at birth, 18 months, 6 years and now 11 years allow us to investigate developmental stability of epigenetic effects over time and assessment of how early-life events and epigenetic state associate with functional and structural differences in neurodevelopmental outcomes in mid-childhood. Our ultimate goal is to identify exposures, events and/or epigenetic variation with value as predictive markers and/or intervention targets to optimize individual neurodevelopmental outcome.

Acknowledgments. We would like to thank all of the supportive families who participated in the PETS study throughout the years: Associate Investigators Charlotte Cecil, David Martino, Jovan Maksimovic, Linda Booij and Richard Tremblay; our amazing research staff, Alicia Clifton, Lada Staskova, Declan Bourke, Matt Bisset, Supriya Raj, Kristal Lau and Dinisha Parmar; our generous volunteers, Jennifer Snowball and Junisha Raj; and the phlebotomists from Royal Children's Hospital Pathology Department.

Financial support. This work was supported by grants from the Australian National Health and Medical Research Council (grant number 1146333 to JMC).

\section{Conflict of interest. None.}

Ethical standards. The authors assert that all procedures contributing to this work comply with the ethical standards of the relevant national and institutional committees on human experimentation and with the Helsinki Declaration of 1975 , as revised in 2008 .

\section{References}

Benjamini, Y., \& Hochberg, Y. (1995). Controlling the false discovery rate: A practical and powerful approach to multiple testing. Journal of the Royal Statistical Society, 57, 289-300.

Bick, J., \& Nelson, C. A. (2016). Early adverse experiences and the developing brain. Neuropsychopharmacology, 41, 177-196.

Carlin, J., Gurrin, L., Sterne, J., Morley, R., \& Dwyer, T. (2005). Regression models for twin studies: A critical review. International Journal Epidemiology, 34, 1089-1099.

Cecil, C. A., Walton, E., Smith, R. G., Viding, E., McCrory, E. J., Relton, C. L., \& Barker, E. D. (2016). DNA methylation and substance-use risk: A prospective, genome-wide study spanning gestation to adolescence. Translational Psychiatry, 6, e976.

Chiarella, J., Tremblay, R. E., Szyf, M., Provencal, N., \& Booij, L. (2015). Impact of early environment on children's mental health: Lessons from DNA methylation studies with monozygotic twins. Twin Research and Human Genetics, 18, 623-634. 
Garton, A., \& Gringart, E. (2005). The development of a scale to measure empathy in 8- and 9-year old children. Australian Journal of Education and Developmental Psychology, 38, 17-25.

Gershon, R. C., Wagster, M. V., Hendrie, H. C., Fox, N. A., Cook, K. F., \& Nowinski, C. J. (2013). NIH toolbox for assessment of neurological and behavioral function. Neurology, 80, S2-S6.

Gordon, L., Joo, J. E., Powell, J. E., Ollikainen, M., Novakovic, B., Li, X., \& Saffery, R. (2012). Neonatal DNA methylation profile in human twins is specified by a complex interplay between intrauterine environmental and genetic factors, subject to tissue-specific influence. Genome Research, 22, 1395-1406.

Hagberg, H., Gressens, P., \& Mallard, C. (2012). Inflammation during fetal and neonatal life: Implications for neurologic and neuropsychiatric disease in children and adults. Annals of Neurology, 71, 444-457.

Heijmans, B. T., Tobi, E. W., Stein, A. D., Putter, H., Blauw, G. J., Susser, E. S., \& Lumey, L. H. (2008). Persistent epigenetic differences associated with prenatal exposure to famine in humans. Proceedings of the National Academy of Sciences of the United States of America, 105, 17046-17049.

Jaffe, A. E., Murakami, P., Lee, H., Fallin, M. D., Feinberg, A. P., \& Irizarry, R. A. (2012). Bump hunting to identify differentially methylated regions in epigenetic epidemiology studies. International Journal of Epidemiology, 41, 200-209.

Levesque, M. L., Fahim, C., Ismaylova, E., Verner, M. P., Casey, K. F., Vitaro, F., \& Booij, L. (2015). The Impact of the in utero and early postnatal environments on grey and white matter volume: A study with adolescent monozygotic twins. Developmental Neuroscience, 37, 489-496.

Li, S., Kim, E., Wong, E. M., Joo, J.-H. E., Nguyen, T. L., Stone, J., \& Hopper, J. (2017). Twin birth changes DNA methylation of subsequent siblings. Scientific Reports, 7, 8463.

Li, S., Wong, E. M., Dugue, P. A., McRae, A. F., Kim, E., Joo, J. E., \& Hopper, J. L. (2018). Genome-wide average DNA methylation is determined in utero. International Journal of Epidemiology, 47, 908-916.

Loke, Y. J., Galati, J. C., Morley, R., Joo, E. J., Novakovic, B., Li, X., \& Craig, J. M. (2013). Association of maternal and nutrient supply line factors with DNA methylation at the imprinted IGF2/H19 locus in multiple tissues of newborn twins. Epigenetics, 8, 1069-1079.

Loke, Y. J., Novakovic, B., Ollikainen, M., Wallace, E. M., Umstad, M. P., Permezel, M., \& Craig, J. M. (2013). The Peri/postnatal Epigenetic Twins Study (PETS). Twin Research and Human Genetics, 16, 13-20.

Makowski, C., Lepage, M., \& Evans, A. C. (2019). Head motion: The dirty little secret of neuroimaging in psychiatry. Journal of Psychiatry \& Neuroscience, 44, 62-68.

Maksimovic, J., Gagnon-Bartsch, J. A., Speed, T. P., \& Oshlack, A. (2015). Removing unwanted variation in a differential methylation analysis of Illumina HumanMethylation450 array data. Nucleic Acids Research, 43, e106.

Manoli, C., Johnson, B., \& Dunlap, R. (2007). Assessing children's environmental worldviews: Modifying and validating the New Ecological Paradigm Scale for use with children. The Journal of Environmental Education, 38, 3-13.

Metz, A. L. (2017). Back to nature: The relationship between nature relatedness on empathy and narcissism in the Millennial Generation. Vistas Online, 11, $1-14$.
Ollikainen, M., Smith, K. R., Joo, E. J., Ng, H. K., Andronikos, R., Novakovic, B., \& Craig, J. M. (2010). DNA methylation analysis of multiple tissues from newborn twins reveals both genetic and intrauterine components to variation in the human neonatal epigenome. Human Molecular Genetics, 19, 4176-4188.

Petanjek, K., \& Kostovic, I. (2012). Epigenetic regulation of fetal brain development and neurocognitive outcome. Proceedings of the National Academy of Sciences of the United States of America, 109, 11062-11063.

Petersen, A., Crockett, L., Richards, M., \& Boxer, A. (1988). A self-report measure of pubertal status: Reliability, validity, and initial norms. Journal of Youth and Adolescence, 17, 117-133.

Phipson, B., Maksimovic, J., \& Oshlak, A. (2016). missMethyl: An R package for analyzing data from Illumina's HumanMethylation450 platform. Bioinformatics 32, 286-288.

Pua, E., Barton, S., Williams, K., Craig, J., \& Seal, M. (2019). Individualised MRI training for paediatric neuroimaging: A child-focused approach. Developmental Cognitive Neuroscience, In Press.

Quach, J., Hiscock, H., Canterford, L., \& Wake, M. (2009). Outcomes of child sleep problems over the school-transition period: Australian population longitudinal study. Pediatrics, 123, 128712-128792.

Reynolds, C., \& Kamphaus, R. (2015). Behavior assessment system for children (3rd ed.) (BASC-3). Bloomington, MN: NCS Pearson.

Roseboom, T., de Rooij, S., \& Painter, R. (2006). The Dutch famine and its long-term consequences for adult health. Early Human Development, 82, 485-491.

Saffery, R., Morley, R., Carlin, J. B., Joo, J. H., Ollikainen, M., Novakovic, B., \& Craig, J. M. (2012). Cohort profile: The peri/post-natal epigenetic twins study. International Journal of Epidemiology, 41, 55-61.

Silva, M., Kilpatrick, N., Craig, J. M., Manton, D., Leong, P., Burgner, D., \& Scurrah, K. (2019). Genetic and early-life environmental influences on dental caries risk: A twin study. Pediatrics, 143, e20183499.

Silva, M. J., Kilpatrick, N. M., Craig, J. M., Manton, D., Leong, P., Burgner, D., \& Scurrah, K. (2019). Etiology of hypomineralized second primary molars: A prospective twin study. Journal of Dental Research, 98, 77-83.

Sung, V., Hiscock, H., Sciberras, E., \& Efron, D. (2008). Sleep problems in children with attention-deficit/hyperactivity disorder: Prevalence and the effect on the child and family. Archives of Pediatrics \& Adolescent Medicine, 162, 336-342.

Temples, H., Willoughby, D., Holaday, B., Rogers, C. R., Wueste, D., Bridges, W., \& Craig, J. M. (2016). Breastfeeding and growth of children in the Peri/postnatal Epigenetic Twins Study (PETS): Theoretical epigenetic mechanisms. Journal of Human Lactation, 32, 481-488.

Veenendaal, M. V., Costello, P. M., Lilllycrop, K. A., de Rooij, S. R., van der Post, J. A., Bossduyt, P. M., \& Roseboom, T. J. (2012). Prenatal famine exposure, health in later life and promotor methylation of four candidate genes. Journal of Developmental Origins of Health and Disease, 3, 450-457.

Wechsler, D. (2016). Wechsler Intelligence Scale for Children (5th ed.) Australian and New Zealand Standardised Edition (WISC-V A\&NZ). Bloomington, MN: NCS Pearson.

Wilkinson, G., \& Robertson, G. (2017). Wide Rande Achievement Test (5th ed.) (WRAT5). Bloomington, MN: NCS Pearson. 